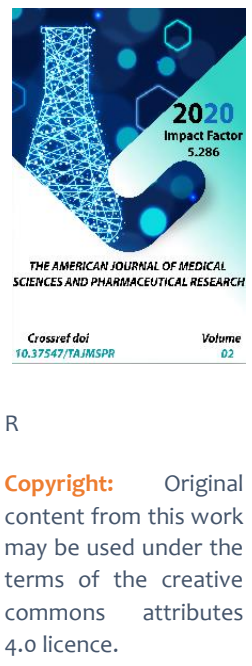

\title{
Oral Sanitation As A Method Of Prevention Of Internal Diseases
}

\author{
Dono Mahmudzhonovna Sapieva \\ Doctor Dentist, Teacher At The Department Of Therapeutic And Orthopedic Stomatology. \\ International Kazakh-Turkish University Named After Hodja Ahmed Yasaui. Kazakhstan
}

Adolat Mominjanovna Abdurakhmanova

Doctor Dentist, Teacher At The Department Of Therapeutic And Orthopedic Stomatology. International Kazakh-Turkish University Named After Hodja Ahmed Yasaui. Kazakhstan.

\section{ABSTRACT}

There are many different ways to examine dental patients, especially those with oral mucositis. In general, they are divided into subjective (question and answer with the patient, questionnaire), objective (seeing the general condition of the patient, the oral cavity). Objective vision is primary and secondary, respectively auxiliary methods.

\section{KEYWORDS}

Stomatitis, catarrhal, acute aphthous ulcers, lip rash, necrotic ulcers, chronic recurrent aphthous ulcers, fibrinosis, necrosis, scarring, glandular, lichenoid forms.

\section{INTRODUCTION}

There are many unique ways to examine dental patients, especially those with oral mucositis. In general, they are divided into subjective (question and answer with the patient, questionnaire), objective (seeing the general condition of the patient, the oral cavity). Objective vision is primary and secondary, respectively auxiliary methods.
The sole purpose of these methods is to make the diagnosis as accurate as possible, and to identify other diseases that have been missed or are occurring at a given time. Hippocrates, Abu Ali Ibn Sino, and later many Russian scholars (G.A. Zaharin, S.P. Botkin, M.Ya. Mudrov, etc.) emphasized the importance of questioning, communicating, and 
interrogating the patient. M.Ya. Mudrov writes: "After a detailed question and answer, in order to determine the general condition of the patient, the doctor should examine him from head to toe, paying attention to the patient's face, eyes, forehead, cheeks, mouth, nose, which is usually may show signs of illness in places ..."

\section{THE MAIN RESULTS AND FINDINGS}

Thus, detailed subjective examinations are important in making a diagnosis. It should be noted that, despite the limited time of the dentist, the examination of these diseases must be thorough and thorough.

The question and answer session with the patient should start from the passport section, without haste: After getting acquainted with the patient's name, age, profession, complaints, when they appeared, approximately (in the opinion of the patient) what they are related to, and so on. it is necessary to know similar information. During the survey, the patient's attention was drawn to additional, goal-oriented questions in order to uncover the symptoms of the presumed disease, to determine when, under what circumstances, and after what the symptoms were associated with, for example, pain. ) and what to relieve and what the dentist should strive to identify.

They usually complain of pain to the dentist. However, sometimes there are various unpleasant sensations in the oral cavity, unpleasant odor, taste disturbances, or various sores, wounds, swelling, bleeding from the gums during tooth extraction, sleep disturbances, low appetite, etc. also patients with many different complaints may come.

If patients present with pain in the oral cavity or in the teeth, gums, tongue, and the like, then what the pain is related to, the time of onset, the causes of the attack, its location, its intensity such as

Or if there is a wound in a certain part of the oral cavity, the time of onset (appearance) of the wound, the cause (in the opinion of the patient), the presence or absence of general diseases of the organism, in the near future The dentist should get acquainted with the diseases and so on. Many symptoms of the disease, such as the patient's inability to speak well, limited movement of the tongue, and pain or inflammation, or the inability to pronounce certain letters, are present during the interview with the patient. , in which changes such as inflammatory processes in the oral cavity, wounds, tumors suggests that the doctor immediately realizes this.

For example, the mucous membrane of the lips or red borders are inflamed, in the presence of pathological changes in them, there is swelling, the movement of the lips (speaking, eating) is accompanied by pain, some letters ( $m, f, b, p, v)$, if this condition is in the mucous membrane of the tongue, the pronunciation of the letters I, s, r, d, s, p, k, is impaired. Or, if there are defects in the palate (jaundice, syphilis, leprosy, trauma), it is known that the pronunciation is distorted.

Thus, the doctor can talk to the patient and at the same time find out about the pathological changes in the organs of the oral cavity and the associated changes in function. As mentioned above, given that the oral cavity is a mirror of the internal organs and systems, the degree of association of symptoms of dental disease with diseases of the internal organs and systems is determined by physician-dentist clinical-laboratory methods, therapists, endocrinologists, psychiatrist is always advisable to use the help of physicians 
working in the ziatr, covenerologist, surgeon, hematologist and other specialties.

After conducting subjective examination methods, it is much more important to switch to objective methods and examine the general condition of the patient, eyes, facial skin, ears, etc., that is, to conduct external objective examinations, observations. allows data collection. In addition to the above, it is necessary to conduct external objective examinations to determine the presence or absence of infections, inflammation, dental diseases, changes in the lymph nodes, changes in the jaw and face, etc.

On external objective examination, the collected tissue was determined by determining the facial and surrounding tissues, the general condition of the patient, facial shape, head position, skin color, eyelid color, speech and pronunciation, and the like. the data will be important in making the diagnosis. Changes in the symmetry of the face, inflammation, swelling, bruising, changes in the bite. Acromegaly, myxedema, Cushing's disease, Quincke's disease, measles, and other similar conditions can cause facial changes. In some diseases of the jaw and face (pulpitis, periodontitis) the color of the cornea changes. When there are various rashes on the face, erythema and other diseases change the color of the skin. Hematoma of the skin in various injuries, stomatitis, lymphadenitis, ulcers (syphilis), leprosy, various skin diseases (seborrhea, cutaneous tuberculosis and other diseases) There may be color changes as well as pronunciations. In diseases of the oral mucosa, it is important to remove the patient and see the whole body.

Additional methods include cytological, histological (biopsy), biochemical and other methods.
See your teeth. The condition of the teeth should also be considered when examining for diseases of the mucous membranes. This is because odontogenic infections, from dental caries to chronic periodontitis, can cause a variety of diseases of the mucous membranes of the dental foci. Determining the number and condition of teeth when they are seen, how they are located in the rows of teeth, the condition of the occlusal teeth, colors, shapes, periodontal tissue, condition of the gums, hypoplasia, the presence of fluorosis, dental anomalies (Getchinson, Furne teeth), it is necessary to get acquainted.

Visualization of the oral mucosa. It is necessary to determine whether there are any exceptions to the norm. Investigation of various changes in the oral cavity in diseases of the gastrointestinal tract, cardiovascular, liver, lungs, urinary tract and other internal organs and systems need to transfer. The test should be performed in natural light. Artificial lamps can change the color of the mucous membrane, which can confuse the doctor. Mucus in acute inflammation, all the signs of inflammation recorded in the pathophysiology of the floor (redness, fever in the site of inflammation, swelling of the tissues, pain and function).

\section{CONCLUSION}

Conclusion is that the disease is caused by a change in the function of an organism - the functions of a particular organ or system - as a result of changes in the environment for a variety of reasons.

In dentistry, when we say "acute pulpitis", "acute periodontitis", "acute osteomyelitis", we mean which organ and how the disease progresses. Of course, these diseases have a general negative effect on the body (sleep, loss of appetite, changes in body 
temperature, fatigue, etc.), even if they occur in the affected, inflamed organs. Each inflammatory process is divided into three stages, although they are characterized by specific symptoms.

1. The initial period.

2. Advanced period.

3. The last period.

In the early stages, each site of inflammation is affected to some extent by the body's resistance and the intensity of microbes and other causes.

A period of advanced, escalating, aggressive inflammation can lead to the development of all adverse effects, leading to dismissal of the patient and loss of ability to work (albeit temporarily). The last period ends with two different outcomes - complete disappearance of the inflammation or the transition to a chronic stage.

\section{REFERENCES}

1. Borovskiy E.V., Danilevskiy N.F. Atlas of diseases of the oral mucosa. - Moscow "Medicine" 1981.

2. Kamilov Kh.P., Ibragimova M.Kh., Mirakhmedova D.Yu. and others. Diseases of the oral mucosa: Uchebnik - Thick: New Age Generation, 2005.

3. Borovskiy E.V. Danilevsky N.F. ATLAS of diseases of the oral mucosa. - Moscow "Medicine" 1991. - 320 p.

4. Banchenko G.V., Maksimov YM. Language is a "mirror" organization. M. Business center "Dentistry" 2000. (Department Library)

5. Borovskiy E.V. "Biology of Politics", M. 2001. (electronic) 3. Barer G.M. Therapeutic dentistry. Part 3. Diseases of the oral mucosa. Moscow. "GEOTARMedia" 2005 (Electronic)
6. 6.Leus P.A., Goreglyad A.A., Chudakova I.O. Diseases of the teeth and mouth stripes. Minsk. "Higher School" 1998 (Electronic)

7. 7 George Laskaris Treatment of diseases of the oral mucosa. Moscow. Ministry of Internal Affairs. 2006 (Department Library)

8. Pachishin M., Got I., Masniy Z. Emergency conditions in dental practice. Lviv. GalDent. 2004 (Electronic)

9. www.med.ru 